\title{
SDF-1/CXCR4 axis induces apoptosis of human degenerative nucleus pulposus cells via the $\mathrm{NF}-\kappa \mathrm{B}$ pathway
}

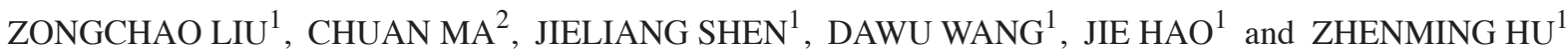 \\ ${ }^{1}$ Department of Orthopedic Surgery, The First Affiliated Hospital of Chongqing Medical University, \\ Chongqing 400016; ${ }^{2}$ Department of Orthopedic Surgery, The Traditional Chinese Medicine \\ Affiliated Hospital of Sichuan Medical University, Luzhou, Sichuan 646000, P.R. China
}

Received July 7, 2015; Accepted May 9, 2016

DOI: $10.3892 / \mathrm{mmr} .2016 .5341$

\begin{abstract}
Intervertebral disc degeneration (IVDD) is a major cause of lower back pain, and increased cell apoptosis is a key characteristic of IVDD. The present study aimed to investigate the effects and mechanism of the stromal cell-derived factor-1 (SDF-1)/C-X-C motif chemokine receptor 4 (CXCR4) axis on apoptosis in human degenerative nucleus pulposus cells (NPCs). The expression levels of SDF-1 and CXCR4 in human intervertebral discs (IVD) were determined using immunohistochemistry and western blot analysis. Apoptosis of primary cultured NPCs was quantified by Annexin V/propidium iodide staining following stimulation with SDF-1 and knockdown of CXCR4 using small interfering RNA (siRNA). The association with the nuclear factor $-\kappa \mathrm{B}(\mathrm{NF}-\kappa \mathrm{B})$ signaling pathway was investigated using CXCR4-siRNA and NF- $\mathrm{NB}$ inhibitor, pyrrolidine dithiocarbamate (PDTC), treatment. The results demonstrated that SDF-1 and its receptor, CXCR4, were upregulated in degenerative IVD samples compared with normal samples. Stimulation with SDF-1 increased the level of apoptosis in cultured NPCs, and conversely, the apoptosis level was suppressed post-transfection with CXCR4 siRNA compared with SDF-1 stimulation alone. Furthermore, SDF-1 treatment increased the level of phosphorylated $\mathrm{NF}-\kappa \mathrm{B}$ subunit P65, which was downregulated following CXCR4 siRNA and PDTC treatment. In addition, CXCR4 siRNA and PDTC inhibited the nuclear translocation of P65, which was induced by SDF-1. Taken together, SDF-1-mediated apoptosis was suppressed by $\mathrm{NF}-\kappa \mathrm{B}$ inhibition using PDTC. In conclusion, the SDF-1/CXCR4 axis promoted cell apoptosis in human degenerative NPCs via the $N F-\kappa B$ pathway, thus suggesting that SDF-1/CXCR signaling may be a therapeutic target for the treatment of degenerative IVD diseases.
\end{abstract}

Correspondence to: Dr Zhenming Hu, Department of Orthopedic Surgery, The First Affiliated Hospital of Chongqing Medical University, 1 Youyi Road, Yuzhong, Chongqing 400016, P.R. China E-mail: spinecenter@163.com

Key words: SDF-1/CXCR4, NF-кB, apoptosis, degenerative intervertebral disc

\section{Introduction}

Lower back pain (LBP) is the sixth most common risk factor for disability worldwide (1), and contributes \$20-100 billion annually in total economic burden (2), which has become a severe socio-economic problem. Effective therapeutic management for LBP remains complex, since the underlying causes are unclear; however, intervertebral disc (IVD) degeneration (IVDD) is the most common diagnosis and target for intervention (3). Among various genetic and/or environmental factors that may contribute to the development of IVDD, cell apoptosis appears to be a major cause (4). A previous investigation confirmed that the number of apoptotic cells in degenerative IVD tissues was significantly increased compared with in normal tissues (5). However, the signal transduction pathways involved in the apoptosis of degenerative IVD cells are not fully understood.

Chemokines are a family of small, soluble chemoattractive cytokines that regulate the recruitment of surrounding responsive cells. In addition, they have previously been reported to affect cell proliferation, apoptosis, differentiation and other physiological activities (6). Stromal cell-derived factor-1 (SDF-1) is one of the most extensively investigated chemokines, which was originally isolated from bone marrow stromal cells and activates various cells by binding to the G-protein-coupled receptor, C-X-C motif chemokine receptor 4 (CXCR4). It has previously been reported that the SDF-1/CXCR4 axis is involved in degradation of the cartilage matrix (7). Furthermore, nuclear factor- $\kappa \mathrm{B}$ $(\mathrm{NF}-\kappa \mathrm{B})$ is a member of a family of transcription factors that is involved in mediating cellular responses to damage, stress and inflammation, and increasing evidence has demonstrated that the NF- $\kappa \mathrm{B}$ signaling pathway is associated with cell apoptosis in nucleus pulposus cells (NPCs) $(8,9)$. However, to the best of our knowledge, the effect of the SDF-1/CXCR4 signaling axis on apoptosis of degenerative NPCs, and whether this effect is mediated through the NF- $\kappa \mathrm{B}$ pathway, has not been previously investigated. On the basis of these observations, the present study aimed to examine the potential regulatory effect of SDF-1/CXCR4 on apoptosis of human NPCs.

\section{Materials and methods}

Primary NPC isolation and culture. Degenerative NP tissues were obtained from seven patients with lumbar disc herniation 
following surgical discectomy. The control group consisted of three patients with lumbar vertebral fracture without previously documented medical history of LBP (Table I). Written informed consent was obtained from all tissue donors and the study protocol was approved by the Ethics Committee of Chongqing Medical University (Chongqing, China). The degree of IVDD was assessed according to Pfirrmann classification by pre-operative magnetic resonance imaging scans (10). Samples from the normal group exhibited Pfirrmann grades I and II, whereas the IVDD group exhibited Pfirrmann grades III-V.

NP tissues from six donors (three per group) were used for immunohistochemistry (IHC) and western blot analysis. NPCs from the degenerative group were isolated by enzymatic digestion and expanded in a monolayer culture model containing Dulbecco's modified Eagle's medium/F 12 (Hyclone; GE Healthcare Life Sciences, Logan, UT, USA) with $10 \%$ (v/v) fetal calf serum (Gibco; Thermo Fisher Scientific, Inc., Waltham, MA, USA), $100 \mu \mathrm{U} / \mathrm{ml}$ penicillin and $100 \mu \mathrm{g} / \mathrm{ml}$ streptomycin, as described previously (11). Cells were incubated at $37^{\circ} \mathrm{C}$ in an atmosphere containing $5 \% \mathrm{CO}_{2}$. Cells at passage III-V were used throughout the in vitro experiments.

IHC staining. NP tissues were fixed in $4 \%$ paraformaldehyde for $24 \mathrm{~h}$, embedded in paraffin and cut serially at $5 \mu \mathrm{m}$ for IHC staining. The IHC staining procedure was performed using a streptavidin-peroxidase immunohistochemical kit (Wuhan Boster Biological Technology, Ltd., Wuhan, China) according to the manufacturer's protocol. Briefly, the tissue sections were incubated with $0.125 \%$ trypsin for $30 \mathrm{~min}$ at $37^{\circ} \mathrm{C}$ for antigen retrieval and then incubated with primary rabbit monoclonal antibodies against SDF-1 (1:100; Abcam, Cambridge, UK) and CXCR4 (1:100; Abcam; cat. no. ab124824) overnight at $4^{\circ} \mathrm{C}$ to stain target protein expression, and immunoglobulin $\mathrm{G}$ (Wuhan Boster Biological Technology, Ltd) was used as a negative control. Finally, sections were incubated in 3-3'-diaminobenzidine (Wuhan Boster Biological Technology, Ltd) to visualize immunoreactivity. Positively stained cells in three different areas were counted under a light microscope (Nikon TS100; Nikon Corporation, Tokyo, Japan).

Cell treatments. Cultured cells were transfected with double-stranded small interfering RNA (siRNA) targeting CXCR4 (Santa Cruz Biotechnology, Inc., Dallas, TX, USA; cat. no. sc-35422) according to the manufacturer's protocol using PepMute siRNA Transfection Reagent (SignaGen Laboratories, Rockville, MD, USA). The cultured cells were transfected with $50 \mathrm{nM}$ siRNA for $72 \mathrm{~h}$, followed by treatment with or without $10 \mathrm{ng} / \mathrm{ml}$ of SDF-1 (Sigma-Aldrich, St. Louis, $\mathrm{MO}, \mathrm{USA}$ ) for $24 \mathrm{~h}$ at $37^{\circ} \mathrm{C}$. In addition, cells were treated with the NF- $\mathrm{NB}$ inhibitor, pyrrolidine dithiocarbamate (PDTC; $20 \mu \mathrm{M}$; Sigma-Aldrich), as described in a previous study (12), for $24 \mathrm{~h}$ to investigate whether SDF-1 regulates the apoptosis of degenerative NPCs via the $\mathrm{NF}-\kappa \mathrm{B}$ pathway.

Reverse transcription-quantitative polymerase chain reaction (RT-qPCR). Total RNA was extracted from the collected tissues and cells, following homogenization with TRIzol (Invitrogen; Thermo Fisher Scientific, Inc.), using RNAiso PLUS kit (Takara Biotechnology Co., Ltd., Dalian, China). RT
Table I. Demographic data of surgical disc samples.

\begin{tabular}{cccccc}
\hline Sample & Gender & Age & Level & Pathology & $\begin{array}{c}\text { Pfirrmann } \\
\text { grade }\end{array}$ \\
\hline 1 & F & 58 & L4/5 & Herniation & III \\
2 & F & 46 & L5/S1 & Herniation & IV \\
3 & M & 65 & L4/5 & Herniation & V \\
4 & F & 39 & L3/4 & Herniation & III \\
5 & F & 31 & L5/S1 & Herniation & III \\
6 & M & 45 & L2/3 & Herniation & IV \\
7 & F & 44 & L4/5 & Herniation & V \\
8 & M & 21 & L1 & Fracture & I \\
9 & M & 39 & L1 & Fracture & I \\
10 & F & 45 & L2 & Fracture & II \\
\hline
\end{tabular}

F, female; M, male.

was performed using $1 \mu \mathrm{g}$ total RNA and random hexamers (Promega Corporation, Madison, WI, USA) to obtain first-strand cDNA. qPCR was performed in a $20 \mu \mathrm{l}$ mixture containing $10 \mu \mathrm{l} \mathrm{SYBR}$ Green Realtime PCR Master Mix (Takara Biotechnology Co., Ltd.), 1.5-3 $\mu \mathrm{l}$ cDNA and 0.4 $\mu \mathrm{l}$ of each primer $(10 \mu \mathrm{M})$. cDNA samples were amplified by qPCR on an ABI Prism 7500 (Applied Biosystems; Thermo Fisher Scientific, Inc.) under the following conditions: $95^{\circ} \mathrm{C}$ for $30 \mathrm{sec} ; 40$ cycles of $95^{\circ} \mathrm{C}$ for $5 \mathrm{sec}, 58^{\circ} \mathrm{C}$ for $5 \mathrm{sec}$ and $72^{\circ} \mathrm{C}$ for $30 \mathrm{sec}$. Glyceraldehyde 3-phosphate dehydrogenase (GAPDH) was used as an internal reference gene and relative expression levels were calculated by the $2^{-\Delta \Delta \mathrm{Cq}}$ method (13). Primer sequences used were as follows: SDF-1, forward 5'-CGTGCT GGTCCTCGTGCTGAC-3', reverse 5'-GCTTTCTCCAGG TACTCCTG-3'; CXCR4, forward 5'-GTCCACGCCACC AACAG-3', reverse 5'-CTGTTGGTGGCGTGGAC-3'; and GAPDH, forward 5'-GCACCGTCAAGGCTGAGAAC-3' and reverse 5'-TGGTGAAGACGCCAGTGGA-3'.

Western blot analysis. Tissues were homogenized and cells were lysed on ice with radioimmunoprecipitation assay lysis buffer in the presence of phenylmethane sulfonyl fluoride (Beyotime Institute of Biotechnology, Haimen, China). Protein concentration was determined using Enhanced BCA Protein assay kit (Beyotime Institute of Biotechnology). Proteins (50 $\mu \mathrm{g})$ were separated by $12 \%$ sodium dodecyl sulfate-polyacrylamide gel electrophoresis. Protein samples were transferred to polyvinylidene difluoride membranes, which were blocked with 5\% nonfat dry milk in Tris-buffered saline (TBS) for $1 \mathrm{~h}$ at room temperature. The membranes were then probed with antibodies against SDF-1 (1:1,000, CXCR4 $(1: 1,000)$, rabbit monoclonal phosphorylated P65 (p-P65; 1:1,000; Cell Signaling Technology, Inc., Danvers, MA, USA; cat. no. 3033), rabbit monoclonal P65 (1:1,000; Cell Signaling Technology, Inc., Danvers, MA, USA; cat. no. 8242) and mouse monoclonal $\beta$-actin (1:8,000; Beyotime Institute of Biotechnology; cat. no. AF0003) overnight at $4^{\circ} \mathrm{C}$. The membrane was washed with TBS and incubated with horseradish peroxidase-conjugated secondary antibody at $37^{\circ} \mathrm{C}$ for $1 \mathrm{~h}$. Following washing with TBS, the membrane 
was visualized with Enhanced Chemiluminescence Plus reagent (Beyotime Institute of Biotechnology) and protein levels were determined using the ChemiDoc XRS+ Imaging system (Bio-Rad Laboratories, Inc., Hercules, CA, USA).

Fluorescence immunocytochemistry. Fluorescence immunocytochemistry was used to detect nuclear translocation of P65. Briefly, cells seeded in 24-well plates were fixed with $4 \%$ paraformaldehyde, washed three times for $10 \mathrm{~min}$ with phosphate-buffered saline, blocked with normal goat serum for $15 \mathrm{~min}$ at room temperature and incubated with P65 antibody (1:100) at $4^{\circ} \mathrm{C}$ overnight. Subsequently, the samples were washed and incubated with a goat anti-mouse fluorescein isothiocyanate-labeled fluorescent secondary antibody (1:500; Beyotime Institute of Biotechnology; cat. no. A0568) at room temperature for $2 \mathrm{~h}$. Nuclear counterstaining was performed with 4',6-diamidino-2-phenylindole (Beyotime Institute of Biotechnology). The cells were imaged at $550 \mathrm{~nm}$ using a fluorescence microscope.

Annexin V/propidium iodide (PI) apoptosis detection. Apoptosis was detected using an Annexin V/propidium iodide (PI) double-staining kit (Nanjing KeyGen Biotech Co., Ltd., Nanjing, China) according to the manufacturer's protocol. Briefly, $1 \times 10^{4}$ cells were collected and suspended in binding buffer, then mixed with $5 \mu \mathrm{l}$ Annexin V and PI. The cells were gently vortexed and incubated at room temperature in the dark for $15 \mathrm{~min}$. Subsequently, $1 \mathrm{X}$ termination buffer was added and flow cytometric analysis on a BD FACScan system (BD Biosciences, Franklin Lakes, NJ, USA) was performed. The apoptotic rate was calculated using FlowJo 8.5.2 software (BD Biosciences) as the sum of the percentage of early (Annexin $\mathrm{V}^{+} / \mathrm{PI}$ ) and late apoptotic cells (Annexin $\mathrm{V}^{+} / \mathrm{PI}^{+}$).

Statistical analysis. All the data are expressed as the mean \pm standard deviation and were analyzed using Prism 5 software (GraphPad Software, Inc., La Jolla, CA, USA). Each experiment was repeated three times. The differences between groups were analyzed by Student's t-test for two groups one-way analysis of variance followed by Tukey's t-test for comparison of multiple groups. $\mathrm{P}<0.05$ was considered to indicate a statistically significant difference.

\section{Results}

Basal SDF-1 and CXCR4 expression levels. IHC staining demonstrated that SDF-1 and CXCR4 were expressed in all donor tissues. SDF-1 was predominantly detected in the extracellular matrix (ECM) and CXCR4 was expressed in the NPCs. The results demonstrated that the SDF-1 integrated optical density values and the percentage of CXCR4-positive cells in the degenerative group was significantly increased compared with the normal group $(\mathrm{P}=0.012$ and $\mathrm{P}<0.001$, respectively; Fig. 1A). Simultaneously, western blotting and PCR analysis confirmed that the expression levels of SDF-1 and CXCR4 were increased in the degenerative group compared with the normal group $(\mathrm{P}=0.018$ and $\mathrm{P}<0.001$, respectively; Fig. 1B). These results suggest that the SDF-1/CXCR4 axis is upregulated in degenerative samples, and therefore may be associated with the IVDD process.
SDF-1/CXCR4 axis regulates NPC apoptosis. To determine the effects of the SDF-1/CXCR4 axis on the apoptosis of degenerative NPCs, cells were treated with $10 \mathrm{ng} / \mathrm{ml} \mathrm{SDF}-1$ for $24 \mathrm{~h}$. qPCR and western blot analysis demonstrated that SDF-1 treatment resulted in an increase in CXCR4 expression at the protein and mRNA levels $(\mathrm{P}=0.034$ and $\mathrm{P}=0.022$, respectively), compared with untreated cells (Fig. 2A). In addition, the results of Annexin V/PI double-staining demonstrated that the number of apoptotic NPCs was significantly increased by SDF-1 treatment compared with untreated cells $(\mathrm{P}<0.001$; Fig. 2B). To further confirm that SDF-1 induces cell apoptosis through its receptor, CXCR4 siRNA was transfected into cells to downregulate the expression of CXCR4 $(\mathrm{P}<0.001$ compared with the control group; Fig. 2A). The results demonstrated that the apoptosis-inducing effects of SDF-1 were reduced following knockdown of endogenous CXCR4 by siRNA compared with SDF-1 treatment only $(\mathrm{P}<0.001$; Fig. $2 \mathrm{~B})$. These results suggest that the SDF-1/CXCR4 axis participates in the positive regulation of apoptosis in degenerative NPCs.

Apoptosis-inducing effects of SDF-1/CXCR4 are mediated via the $N F-\kappa B$ pathway. The $N F-\kappa B$ signaling pathway has previously been demonstrated to regulate cell survival and apoptosis (14). To investigate the association between $\mathrm{NF}-\kappa \mathrm{B}$ and SDF-1/CXCR4 signaling, the present study examined the effect of SDF-1 treatment on the phosphorylation of $\mathrm{NF}-\kappa \mathrm{B}$ subunit P65. The levels of p-P65 were markedly increased in NPCs stimulated with SDF-1 compared with the control cells $(\mathrm{P}=0.008$; Fig. 3A). Furthermore, treating NPCs with $\mathrm{NF}-\kappa \mathrm{B}$ inhibitor, PDTC, or CXCR4 siRNA markedly reduced the level of p-P65 (Fig. 3A). Furthermore, the cells were subjected to immunofluorescence to analyze the nuclear translocation of P65. As demonstrated in Fig. 3B, PDTC and CXCR4-siRNA treatment inhibited the nuclear translocation of $\mathrm{P} 65$ compared with SDF-1 treatment. To determine the effect of P65 activation on SDF-1-induced cell apoptosis, NPCs were treated with $20 \mu \mathrm{M}$ PDTC for $24 \mathrm{~h}$, followed by incubation with $10 \mathrm{ng} / \mathrm{ml}$ SDF-1 for an additional $24 \mathrm{~h}$. Annexin V/PI apoptosis detection analysis demonstrated that, compared with SDF-1 treatment, inhibition of $\mathrm{P} 65$ activation by PDTC significantly reduced the SDF-1-induced increase in NPC apoptosis ( $\mathrm{P}=0.029$; Fig. 3C). These results indicate that the apoptosis-inducing effects of the SDF-1/CXCR4 axis are mediated via NF- $\mathrm{KB}$ signaling.

\section{Discussion}

The present study demonstrated that the expression levels of SDF-1 and CXCR4 are significantly increased in degenerative IVD tissues compared with normal NP tissues. Therefore, it was hypothesized that the SDF-1/CXCR4 signaling axis may be involved in IVDD. To verify this hypothesis, cultured degenerative NPCs were treated with SDF-1 following knockdown of CXCR4 using siRNA. To the best of our knowledge, the present study is the first to report that the SDF-1/CXCR4 axis accelerates apoptosis in degenerative NPCs. Furthermore, the number of apoptotic NPCs was significantly decreased following inhibition of NF- $\kappa \mathrm{B}$ activation using PDTC, which suggests the apoptosis-inducing effect of the SDF-1/CXCR4 axis in degenerative NPCs is mediated via $\mathrm{NF}-\kappa \mathrm{B}$ signaling. 
A
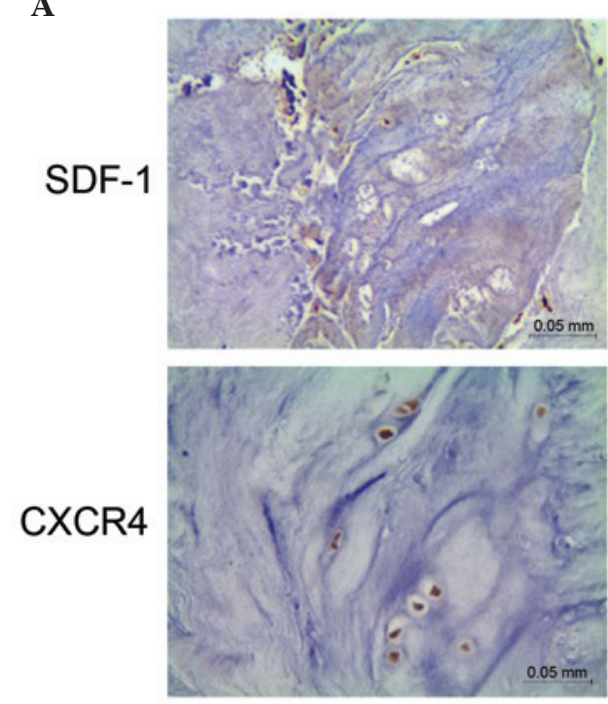

B
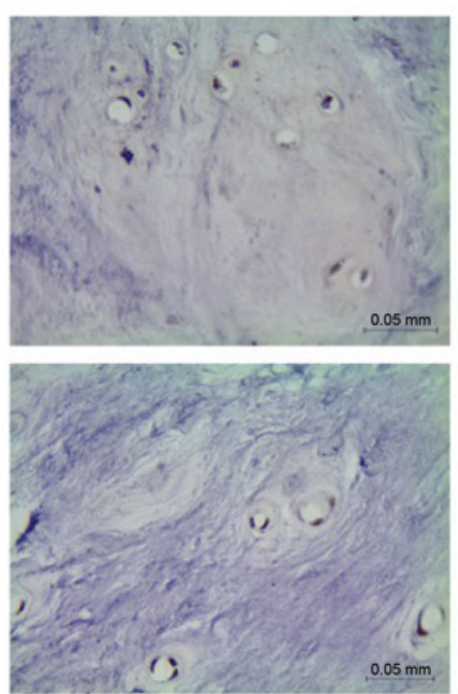

Normal group
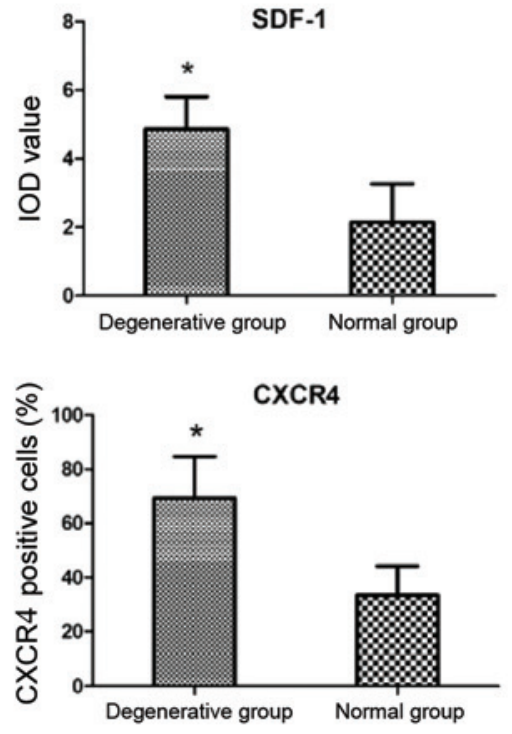

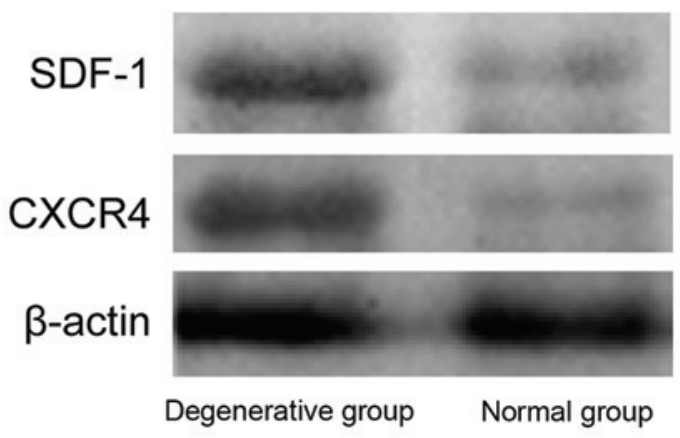

$11 \mathrm{kDa}$

$39 \mathrm{kDa}$

$42 \mathrm{kDa}$

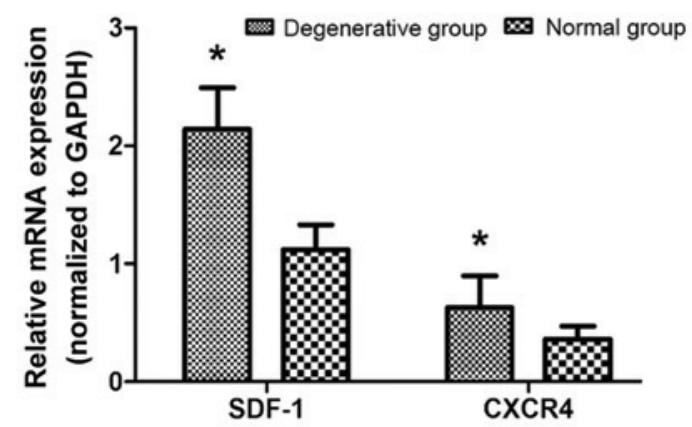

Figure 1. Analysis of SDF-1 and CXCR4 expression in NP tissues from normal and degenerative groups. (A) SDF-1 and CXCR4 were determined by immunohistochemical staining, and SDF-1 was predominantly observed in the extracellular matrix, whereas CXCR4 was expresssed in NP cells. Magnification, x200. (B) SDF-1 and CXCR4 expression levels in untreated NP tissues were determined by western blot analysis and reverse transcription-quantitative polymerase chain reaction. Data are presented as the mean \pm standard deviation from three independent experiments. * $\mathrm{P}<0.05$ vs. the normal control group. NP, nucleus pulposus; SDF-1, stromal cell-derived factor-1; CXCR4, C-X-C motif chemokine receptor 4; GAPDH, glyceraldehyde 3-phosphate dehydrogenase; IOD, integrated optical density.

It is well established that NPCs are involved in resistance against mechanical loading, with the synthesis of ECM important to maintain spinal stability. Therefore, loss of NPCs has been demonstrated to be correlated with the pathological process of IVDD (15). Ahsan et al (16) analyzed the molecular and morphological features of 32 herniated disc specimens and four control disc samples, demonstrating that there was increased caspase-3 activity and apoptotic-positive stained DNA fragments in the degenerative disc samples. Furthermore, electron microscopy findings suggested that there was enhanced programmed cell death in the degenerative discs (16). In addition, a previous study indicated that the percentage of apoptotic cells in degenerative NP specimens was significantly increased compared with normal controls, as demonstrated by terminal deoxynucleotidyl transferase dUTP nick end labeling staining (5). However, the factors that induce NPC apoptosis during the IVDD process have not been fully elucidated.

IVDD is characterized by an increase in the expression levels of proinflammatory cytokines, including tumor necrosis factor- $\alpha$ and interleukin (IL)-1 $\beta$, which induce ECM degradation, chemokine production and changes in cell phenotype (17). The release of chemokines promotes the infiltration and activation of immune cells, which amplifies the inflammatory cascade. SDF-1 is highly expressed in inflamed tissues, where it attracts activated $\mathrm{CXCR} 4^{+} \mathrm{T}$ cells, thus enhancing local inflammatory responses (18). The SDF-1/CXCR4 axis has previously been associated with the pathogenesis of chronic inflammatory diseases, including osteoarthritis (19) and rheumatoid arthritis (20). The current study focused on the differential expression of SDF-1 and CXCR4 in normal and degenerative IVD tissues. The results demonstrated that the expression levels of SDF-1 and CXCR4 were increased in degenerative IVD tissues. The SDF-1/CXCR4 axis has previously been reported to have various targets, via which it stimulates chondrocyte proliferation, differentiation and apoptosis $(21,22)$. Therefore, it was hypothesized that the SDF-1/CXCR4 axis is also involved in the IVDD process. In support of this, the present study demonstrated that the number of apoptotic NPCs was significantly increased following SDF-1 stimulation, and this apoptosis-inducing effect was inhibited by siRNA-mediated silencing of CXCR4 expression. 
A

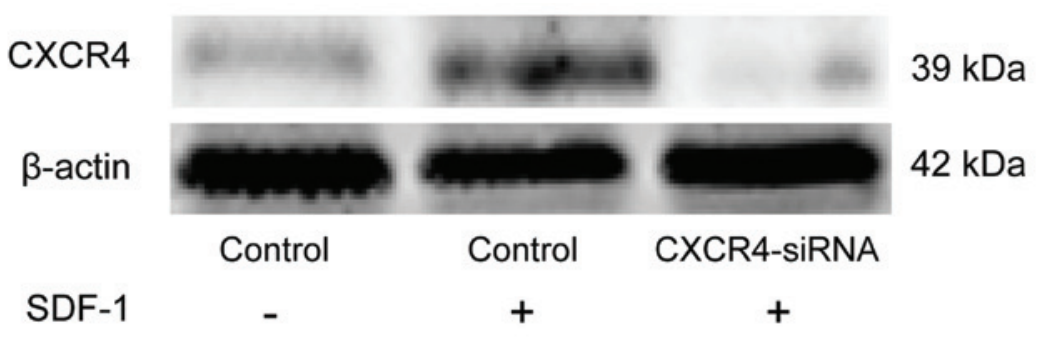

\section{CXCR4}

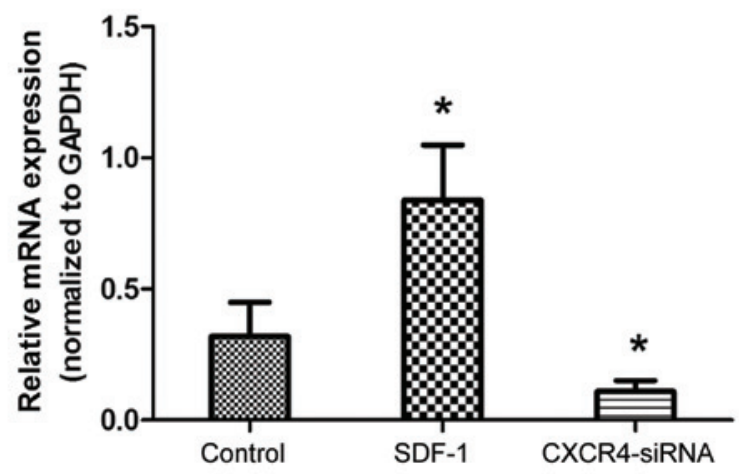

$\mathbf{B}$

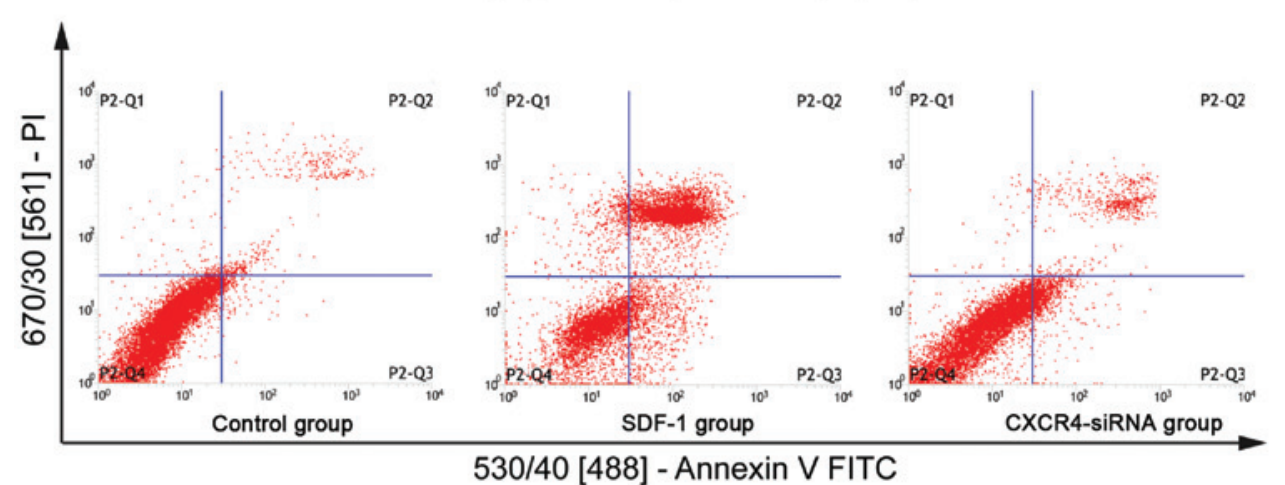

530/40 [488] - Annexin V FITC

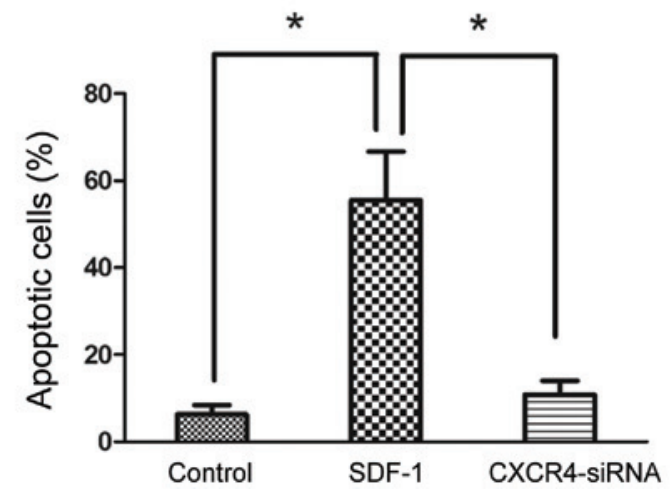

Figure 2. SDF-1/CXCR4 axis induces apoptosis of degenerative NP cells. (A) NP cells were treated with SDF-1 and siRNA targeting CXCR4, the protein and mRNA expression levels of CXCR4 were assessed by western blotting and reverse transcription-quantitative polymerase chain reaction. "P $<0.05$ vs. the control group. (B) Cell apoptotic percentage were measured by Annexin V/PI double-staining. ${ }^{*} \mathrm{P}<0.05$ vs. the SDF-1 group. Data are presented as the mean \pm standard deviation from three independent experiments. NP, nucleus pulposus; SDF-1, stromal cell-derived factor-1; CXCR4, C-X-C motif chemokine receptor 4; siRNA, small interfering RNA; PI, propidium iodide; FITC, fluorescein isothiocyanate; GAPDH, glyceraldehyde 3-phosphate dehydrogenase.

These results clearly indicated that there is a positive correlation between SDF-1/CXCR4 expression and cell apoptosis in IVDD.

$\mathrm{NF}-\kappa \mathrm{B}$ is a rapidly inducible transcription factor, which regulates the expression of numerous genes to mediate various cellular processes, including cell apoptosis, survival and the immune response (23). It has previously been demonstrated that the NF- $\kappa \mathrm{B}$ signaling pathway is involved in IL- $1 \beta$-induced chondrocyte apoptosis (24). Regarding the inflammatory mechanisms of $\mathrm{NF}-\kappa \mathrm{B}$, a previous study reported that the $N F-\kappa B$ pathway is associated with the release of proinflammatory factors (25). Furthermore, SDF-1 is reported to be an 
A

p-P65

$\beta$-actin

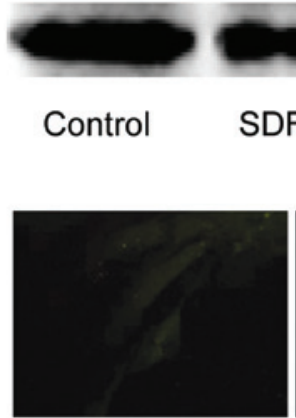

B

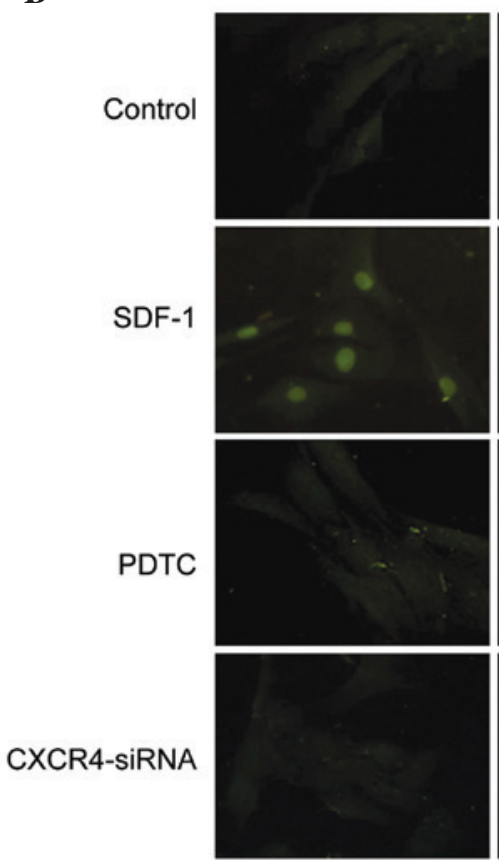

P65

C
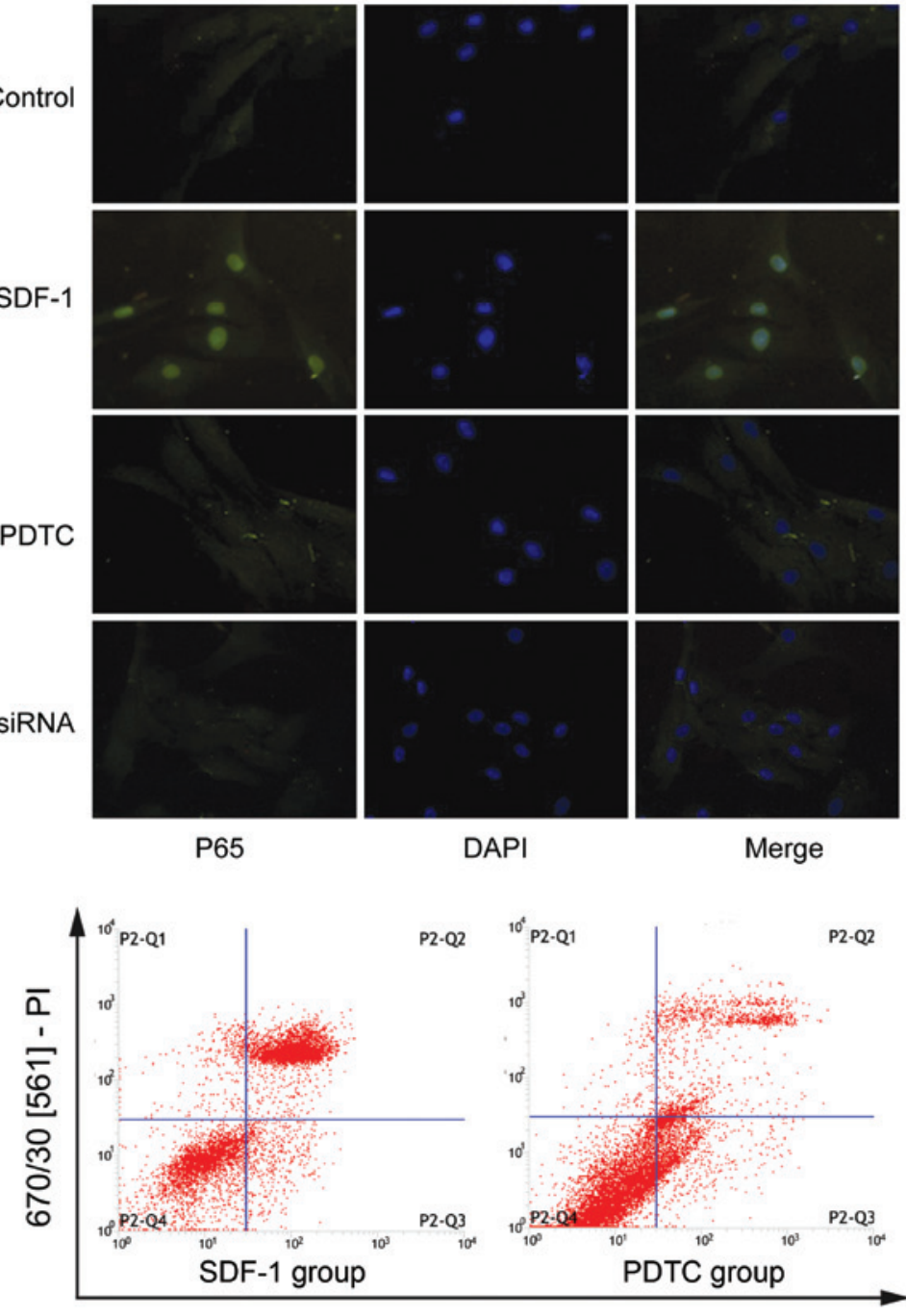

DAPI

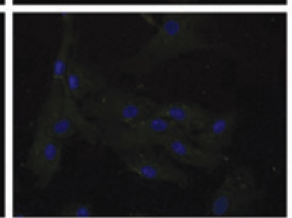

Merge

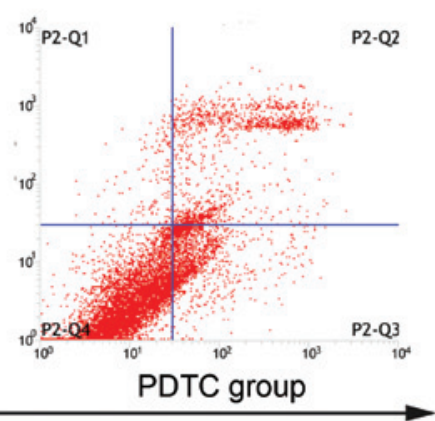

530/40 [488] - Annexin V FITC

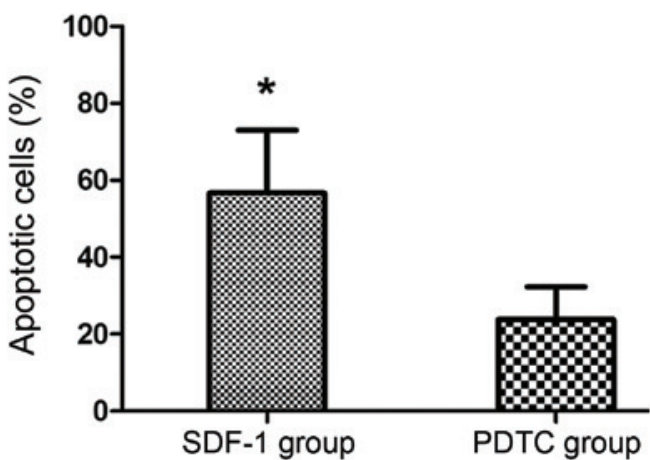

Figure 3. SDF-1/CXCR4 axis induces cell apoptosis via the NF-kB pathway. (A) NP cells were treated with SDF-1, NF-kB inhibitor PDTC and CXCR4-siRNA, and the phosphorylation level of NF-kB subunit P65 was detected by western blotting. (B) Fluorescence immunocytochemistry was used to detect nuclear translocation of P65. (C) To observe the effect of NF-kB on SDF-1-induced cell apoptosis, NP cells were analyzed by flow cytometry following treatment with PDTC. Data are presented as the mean \pm standard deviation from three independent experiments. ${ }^{*} \mathrm{P}<0.05$ vs. the PDTC group. NF- $\kappa \mathrm{B}$, nuclear factor- $\kappa \mathrm{B}$; NP, nucleus pulposus; SDF-1, stromal cell-derived factor-1; PDTC, pyrrolidine dithiocarbamate; CXCR4, C-X-C motif chemokine receptor 4; siRNA, small interfering RNA; DAPI, 4',6-diamidino-2-phenylindole; PI, propidium iodide; FITC, fluorescein isothiocyanate. 
important chemokine during the immune response; however, the molecular interaction between the SDF-1/CXCR4 axis and $\mathrm{NF}-\kappa \mathrm{B}$ in degenerative NPCs remains unclear. The current study demonstrated that treatment with the NF- $\kappa \mathrm{B}$ inhibitor, PDTC, inhibited SDF-1-induced NPC apoptosis in vitro, and suppressed P65 phosphorylation and nucleus translocation, indicating that $\mathrm{NF}-\kappa \mathrm{B}$-dependent signaling is involved in regulation of cell apoptosis by SDF-1 in degenerative NPCs.

A limitation of the current study is that in vitro cultured monolayers of NPCs were used. A previous study demonstrated that NPCs easily lose their phenotype when cultured in vitro as a monolayer (26). The use of a $3 \mathrm{D}$ alginate culture of NPCs was considered for the present study; however, the preliminary testing demonstrated a low siRNA transfection efficiency when using an alginate scaffold for 3D culture. Therefore, the experiments were performed using monolayer culture to improve the transfection efficiency. Furthermore, to elucidate the association between the SDF-1/CXCR4 axis and NPC apoptosis, further research is required using in vivo animal models, and the activation state of upstream and downstream molecules of the NF- $\mathrm{B}$ signaling pathway should be examined.

In conclusion, the current study demonstrated that the expression levels of SDF-1 and CXCR4 were increased in degenerative NP tissues. Knockdown of CXCR4, an SDF-1 receptor, reduced the number of apoptotic degenerative NPCs. Notably, activation of NF- $\kappa$ B subunit P65 was associated with the apoptosis-inducing effect of SDF-1. Therefore, the SDF-1/CXCR4 axis is considered to induce apoptosis of human degenerative NPCs via the NF- $\kappa \mathrm{B}$ signaling pathway. The results of the present study suggest that the SDF-1/CXCR4 axis may be a therapeutic target for the treatment of degenerative disc disease.

\section{Acknowledgements}

This study was supported by grants from the National Natural Science Foundation of China (grant nos. 81171751 and 81372003).

\section{References}

1. Murray CJ, Vos T, Lozano R, Naghavi M, Flaxman AD, Michaud C, Ezzati M, Shibuya K, Salomon JA, Abdalla S, et al: Disability-adjusted life years (DALYs) for 291 diseases and injuries in 21 regions, 1990-2010: A systematic analysis for the Global Burden of Disease Study 2010. Lancet 380: 2197-2223, 2012.

2. Parthan A, Evans CJ and Le K: Chronic low back pain: Epidemiology, economic burden and patient-reported outcomes in the USA. Expert Rev Pharmacoecon Outcomes Res 6: 359-369, 2006.

3. de Schepper EI, Damen J, van Meurs JB, Ginai AZ, Popham M, Hofman A, Koes BW and Bierma-Zeinstra SM: The association between lumbar disc degeneration and low back pain: The influence of age, gender, and individual radiographic features. Spine (Phila Pa 1976) 35: 531-536, 2010.

4. Ding F, Shao ZW and Xiong LM: Cell death in intervertebral disc degeneration. Apoptosis 18: 777-785, 2013.

5. Wang D, Hu Z, Hao J, He B, Gan Q, Zhong X, Zhang X, Shen J, Fang J and Jiang W: SIRT1 inhibits apoptosis of degenerative human disc nucleus pulposus cells through activation of Akt pathway. Age (Dordr) 35: 1741-1753, 2013.
6. Pulsatelli L, Dolzani P, Piacentini A, Silvestri T, Ruggeri R, Gualtieri G, Meliconi R and Facchini A: Chemokine production by human chondrocytes. J Rheumatol 26: 1992-2001, 1999.

7. Kanbe K, Takagishi K and Chen Q: Stimulation of matrix metalloprotease 3 release from human chondrocytes by the interaction of stromal cell-derived factor 1 and CXC chemokine receptor 4. Arthritis Rheum 46: 130-137, 2002.

8. Wang XH, Hong X, Zhu L, Wang YT, Bao JP, Liu L, Wang F and Wu XT: Tumor necrosis factor alpha promotes the proliferation of human nucleus pulposus cells via nuclear factor- $\kappa \mathrm{B}, \mathrm{c}-\mathrm{Jun}$ $\mathrm{N}$-terminal kinase, and p38 mitogen-activated protein kinase. Exp Biol Med (Maywood) 240: 411-417, 2015.

9. Zhongyi S, Sai Z, Chao L and Jiwei T: Effects of nuclear factor kappa B signaling pathway in human intervertebral disc degeneration. Spine (Phila Pa 1976) 40: 224-232, 2015.

10. Pfirrmann CW, Metzdorf A, Zanetti M, Hodler J and Boos N: Magnetic resonance classification of lumbar intervertebral disc degeneration. Spine (Phila Pa 1976) 26: 1873-1878, 2001.

11. Zhang Z, Kakutani K, Maeno K, Takada T, Yurube T, Doita M, Kurosaka M and Nishida K: Expression of silent mating type information regulator 2 homolog 1 and its role in human intervertebral disc cell homeostasis. Arthritis Res Ther 13: R200, 2011.

12. Zhou H, Sheng L, Wang H, Xie H, Mu Y, Wang T and Yan J: Anti- $\beta 2$ GPI/ $\beta 2$ GPI stimulates activation of THP-1 cells through TLR4/MD-2/MyD88 and NF- $\mathrm{B}$ signaling pathways. Thromb Res 132: 742-749, 2013.

13. Livak KJ and Schmittgen TD: Analysis of relative gene expression data using real-time quantitative PCR and the 2(-Delta Delta C(T)) Method. Methods 25: 402-408, 2001.

14. Shen J, Fang J, Hao J, Zhong X, Wang D, Ren H and Hu Z: SIRT1 inhibits the catabolic effect of IL- $1 \beta$ through TLR2/SIRT1/NF- $\mathrm{B}$ pathway in human degenerative nucleus pulposus cells. Pain Physician 19: E215-E226, 2016.

15. Wang SZ, Rui YF, Lu J and Wang C: Cell and molecular biology of intervertebral disc degeneration: Current understanding and implications for potential therapeutic strategies. Cell Prolif 47: 381-390, 2014.

16. Ahsan R, Tajima N, Chosa E, Sugamata M, Sumida M and Hamada M: Biochemical and morphological changes in herniated human intervertebral disc. J Orthop Sci 6: 510-518, 2001.

17. Risbud MV and Shapiro IM: Role of cytokines in intervertebral disc degeneration: Pain and disc content. Nat Rev Rheumatol 10: 44-56, 2014.

18. Palomino DC and Marti LC: Chemokines and immunity. Einstein (Sao Paulo) 13: 469-473, 2015.

19. Wei F, Moore DC, Wei L, Li Y,Zhang G, Wei X, Lee JK and Chen Q: Attenuation of osteoarthritis via blockade of the SDF-1/CXCR4 signaling pathway. Arthritis Res Ther 14: R177, 2012.

20. Kim HR, Kim KW, Kim BM, Jung HG, Cho ML and Lee SH: Reciprocal activation of CD4+ T cells and synovial fibroblasts by stromal cell-derived factor 1 promotes RANKL expression and osteoclastogenesis in rheumatoid arthritis. Arthritis Rheumatol 66: 538-548, 2014.

21. Sukegawa A, Iwasaki N, Kasahara Y, Onodera T, Igarashi T and Minami A: Repair of rabbit osteochondral defects by an acellular technique with an ultrapurified alginate gel containing stromal cell-derived factor-1. Tissue Eng Part A 18: 934-945, 2012.

22. Wei L, Sun X, Kanbe K, Wang Z, Sun C, Terek R and Chen Q: Chondrocyte death induced by pathological concentration of chemokine stromal cell-derived factor-1. J Rheumatol 33: 1818-1826, 2006.

23. Lee H, Jeon J, Ryu YS, Jeong JE, Shin S, Zhang T, Kang SW, Hong JH and Hur GM: Disruption of microtubules sensitizes the DNA damage-induced apoptosis through inhibiting nuclear factor $\kappa \mathrm{B}(\mathrm{NF}-\kappa \mathrm{B}) \mathrm{DNA}-$ binding activity. J Korean Med Sci 25: 1574-1581, 2010.

24. Zhang X, Xu X, Xu T and Qin S: $\beta$-Ecdysterone suppresses interleukin-1 $\beta$-induced apoptosis and inflammation in rat chondrocytes via inhibition of NF- $\kappa$ B signaling pathway. Drug Dev Res 75: 195-201, 2014.

25. Roman-Blas JA and Jimenez SA: NF-kappaB as a potential therapeutic target in osteoarthritis and rheumatoid arthritis. Osteoarthritis Cartilage 14: 839-848, 2006.

26. Kluba T, Niemeyer T, Gaissmaier $C$ and Gründer T: Human anulus fibrosis and nucleus pulposus cells of the intervertebral disc: Effect of degeneration and culture system on cell phenotype. Spine (Phila Pa 1976) 30: 2743-2748, 2005. 